\title{
Volumetric laser endomicroscopy in Barrett's esophagus: ready for primetime
}

\author{
Trevor Houston ${ }^{1}$, Prateek Sharma ${ }^{2}$ \\ ${ }^{1}$ Department of Internal Medicine, University of Nevada, Las Vegas School of Medicine, Las Vegas, NV, USA; ${ }^{2}$ Department of Gastroenterology, \\ University of Kansas Medical Center, Kansas City, KS, USA \\ Contributions: (I) Conception and design: T Houston, P Sharma; (II) Administrative support: None; (III) Provision of study material or patients: \\ None; (IV) Collection and assembly of data: None; (V) Data analysis and interpretation: T Houston, P Sharma; (VI) Manuscript writing: All authors; \\ (VII) Final approval of manuscript: All authors. \\ Correspondence to: Prateek Sharma. Department of Gastroenterology and Hepatology, The University of Kansas Medical Center, 3901 Rainbow Blvd., \\ Kansas City, KS, USA. Email: psharma@kumc.edu.
}

\begin{abstract}
Barrett's esophagus (BE) is the condition where intestinal metaplastic changes are found in the normal stratified squamous epithelium of the esophagus predisposing an individual to dysplasia and esophageal adenocarcinoma (EAC). It tends to affect males and is often the result of chronic gastroesophageal reflux disease (GERD). The current standard of therapy for diagnosing Barrett's is white light endoscopy (WLE) with biopsies obtained using the Seattle protocol. Multiple newer advanced modalities have been developed to improve diagnostic abilities, including volumetric laser endomicroscopy (VLE). This technique utilizes second generation optical coherence tomography (OCT) to provide an enhanced circumferential image to a depth of $3 \mathrm{~mm}$ with the potential for improved diagnostic yield for dysplasia, particularly submucosal lesions or lesions not seen by WLE. It has also been evaluated in guiding mapping of endotherapy as well as post therapy surveillance for recurrence. Although the results have been promising when used with current diagnostic standards, overall data are limited to support the routine use of VLE.
\end{abstract}

Keywords: Barrett's; dysplasia; endomicroscopy; esophagus

Received: 11 September 2019; Accepted: 14 November 2019; Published: 05 April 2020.

doi: $10.21037 / \operatorname{tgh} .2019 .11 .16$

View this article at: http://dx.doi.org/10.21037/tgh.2019.11.16

\section{What is Barrett's esophagus (BE)?}

$\mathrm{BE}$ is a condition in which metaplastic columnar epithelium with both gastric and intestinal features replaces the stratified squamous epithelium normally lining the esophagus. BE develops as a result of chronic gastroesophageal reflux disease (GERD) and predisposes to development of adenocarcinoma of the esophagus (EAC) by a risk of 40 to 50 fold over the general population. It is estimated that $5.6 \%$ of American Adults have BE, however many go undiagnosed (1). A 2003 study from the University of Indiana with 961 patients undergoing EGD and colonoscopy found that the prevalence was $6.8 \%$. Among 556 patients that denied GERD symptoms, the incidence was $5.6 \%$. Of the 384 patients that reported GERD symptoms, the incidence was $8.3 \%$, most being of the short segment type (2).

After a columnar lined esophagus $>1 \mathrm{~cm}$ is seen on endoscopy, biopsies will assist in classifying as non-dysplastic, indefinite for dysplasia, dysplastic or EAC. Dysplasia can further be characterized as low grade (LGD), high grade (HGD), or intramucosal carcinoma (IMC). Estimates of annual incidence of cancer in $\mathrm{BE}$ varies from $0.1 \%$ to $0.4 \%$ based on available studies suggesting that the rate of progression for the general population with $\mathrm{BE}$ is $0.25 \%$ per year, a rate of $0.54 \%$ per year for LGD and as high as $4-8 \%$ per year in HGD (3-5). Once a diagnosis of BE is established treatment modality is based on the presence and degree of dysplasia, in addition to indefinite proton pump inhibitor therapy and ACG recommended surveillance. 
Non-dysplastic BE can typically have surveillance endoscopy every 3-5 years (6). Endoscopic treatment typically starts when HGD or early EAC is seen and initially treated with endoscopic resection techniques, such as resection cap, multi band mucosectomy, or submucosal dissection, followed by RFA or hybrid argon plasma coagulation (7-13). RFA can be used on HGD dysplasia and IMC after endoscopic mucosal resection, however, most patients undergoing RFA will have flat or non-visible HGD, or LGD. The use of RFA in nondysplastic $\mathrm{BE}$ is controversial as the risk of cancer progression in these patients is extremely low and thus should be avoided (3-5). Esophagectomy is usually reserved for Invasive EAC and can carry a significant morbidity, ranging from $4.9 \%$ to $12.1 \%$ based on current studies (14).

\section{How to screen and survey in BE patients?}

Screening for BE can be considered in men with chronic or frequent GERD who have additional risk factors including age over 50, white race, hiatal hernia, elevated body mass index and intra-abdominal distribution of fat (6). The current gold standard diagnosis of BE starts with upper endoscopy with visualization under white light endoscopy (WLE), where pink or salmon colored mucosa extending for $>1 \mathrm{~cm}$ above the GEJ will typically be seen in comparison to normal squamous mucosa. The extent of metaplastic change is then described using the Prague $C \& M$ classification in addition to the location of the diaphragmatic pinch, gastroesophageal junction (GEJ), and squamocolumnar junction $(15,16)$. Biopsies are obtained to determine presence of dysplasia for further progression to EAC. The Seattle Protocol is the method used where 4 quadrant biopsy specimens are obtained every $2 \mathrm{~cm}$ intervals in those without mucosal irregularities or prior dysplasia and $1 \mathrm{~cm}$ in those with irregularities or prior dysplasia $(15,17)$. This is in addition to targeted biopsy sampling of visible abnormalities.

Dysplasia may not be apparent during inspection with WLE. As this protocol is not without flaws, missed disease can occur, with estimated $25.3 \%$ of procedures related to esophageal adenocarcinoma (EAC) occurring within one year of surveillance endoscopy (18). Current guidelines suggest the use of high definition WLE when detecting dysplastic lesions as evidence shows its superiority to standard definition $(15,19,20)$. In addition, as the $\mathrm{BE}$ segment increases, the time needed for proper surveillance does as well. Longer inspection times have shown to increase detection of dysplasia $(15,21,22)$. One study shows adherence in the community to the Seattle protocol decreases as the $\mathrm{BE}$ segment gets longer (23). One response to the increased surveillance time needed was wide-area epithelial sampling (WATS.) Rather than obtaining a discreet number of forceps biopsies per quadrant, WATS uses an abrasive brush to circumferentially sweep the esophagus to yield samples of the surface and deeper layers that are then analyzed by a computer $(15,24)$.

Other advanced modalities allow targeted biopsies to be obtained, rather than random. These include narrow band imaging (NBI), Chromoendoscopy and Confocal laser endomicroscopy (CLE.) NBI uses the blue-green spectrum of light $(415-540 \mathrm{~nm})$ to capitalize on peak absorption of hemoglobin accentuating visualization of mucosal vasculature. The field of view is $140^{\circ}$ with a depth of $3-100 \mathrm{~mm}(15,21)$. Chromoendoscopy is the use of absorptive and contrast stains applied to esophageal mucosa to highlight superficial mucosal architecture and is used in conjunction with WLE (15). The equipment needed is widely available and the stains are generally inexpensive and safe. CLE utilizes fluorescence emission by a low powered laser that can generate in vivo images of esophageal mucosa at histologic-level resolution. This can be endoscope based (eCLE) with a field of view of $475 \mu \mathrm{m}$ or probe based (pCLE) with a field of view of 240-600 $\mu \mathrm{m}$ (15).

The Preservation and Incorporation of Valuable Endoscopic Innovations Committee (PIVI) of the ASGE developed performance thresholds for using image assisted targeted biopsies during surveillance as $90 \%$ sensitivity, $80 \%$ Specificity and negative predictive value of $98 \%$ for detecting HGD or EAC (25). Of the newer modalities, only NBI, eCLE and chromoendoscopy with acetic acid have been recommended based on these performance thresholds $(26,27)$.

\section{What is volumetric laser endomicroscopy (VLE)?}

VLE is a newer endoscopic imaging modality using optical coherence tomography (OCT). VLE specifically uses second generation OCT technology called optical frequency domain imaging, in a balloon based system that was developed to address slower processing time and limited scanning area of traditional OCT devices $(15,28,29)$ (Figure 1). Infrared light generates a circumferential scan of $6 \mathrm{~cm}$ segments of esophagus to a depth of $3 \mathrm{~mm}$ allowing for visualization of esophageal layers and submucosal vascular networks with $7 \mu \mathrm{m}$ axial resolution in a much shortened processing time, typically $60-90$ seconds $(30,31)$.

$\mathrm{VLE}$ in BE patients has been evaluated in surveillance 


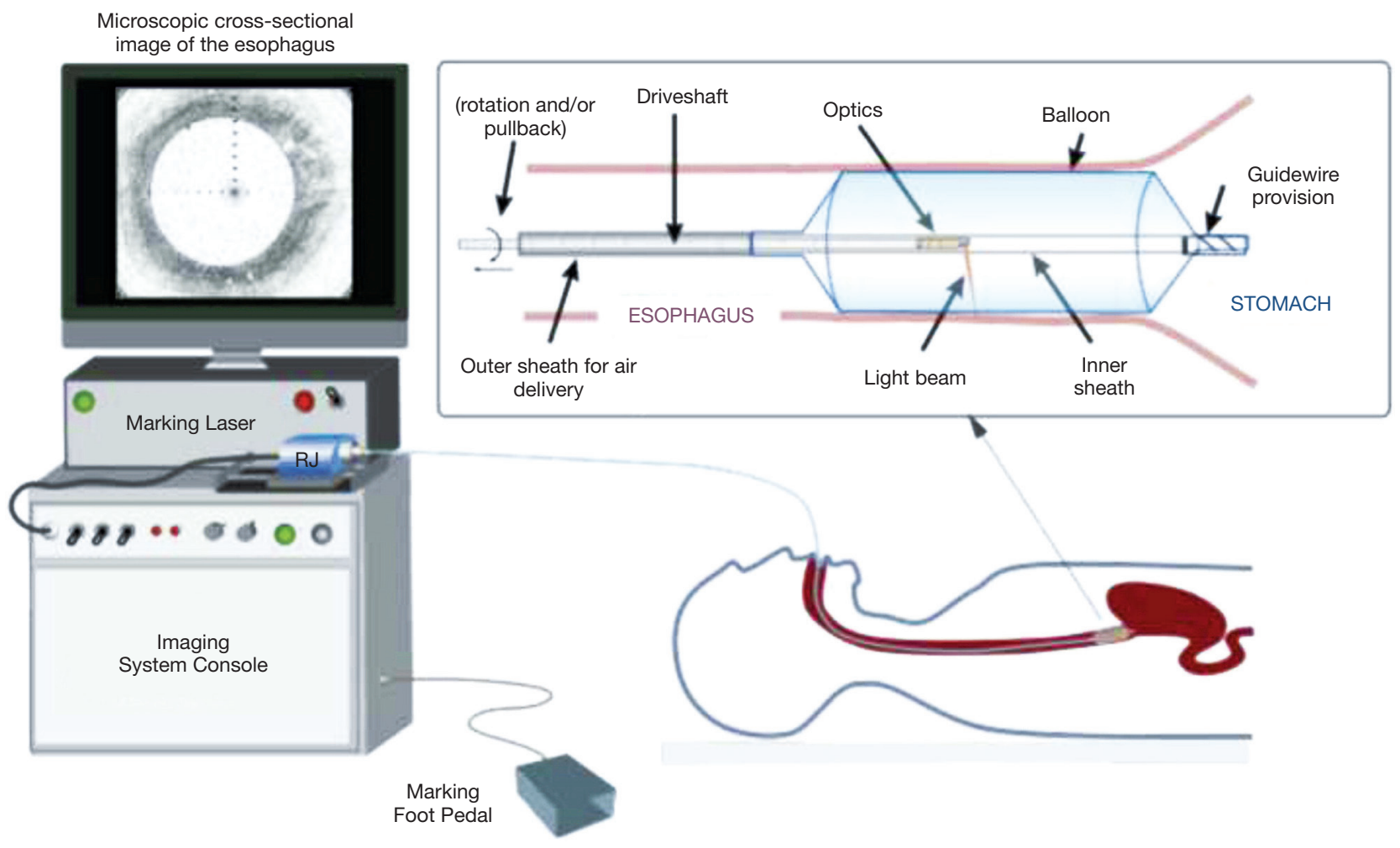

Figure 1 Schematic of the VLE-guided biopsy system and balloon-centering catheter. The interchangeable balloon catheter is inserted into the esophagus at the gastroesophageal junction and inflated. The balloon catheter is connected to the imaging system via an optical rotary junction. The rotary junction spins a driveshaft that encloses an optical fiber. The optical fiber is terminated by focusing optics at the distal end that spin with the driveshaft. The driveshaft is pulled back while spinning to effectuate a helical OCT scan of the esophagus. A foot pedal is used to initiate laser marking. The entire procedure is monitored by real-time visualization of the displayed, cross-sectional OCT image. VLE, volumetric laser endomicroscopy; OCT, optical coherence tomography. (Figure used with permission) (29).

of high risk, treatment naive patients, to assist in treatment selection of those with previously detected precancerous or cancerous lesions, and in surveillance post treatment. VLE has been used in staging early squamous cell carcinoma of the esophagus as well as to guide endoscopic myotomy for treatment of achalasia. A smaller version of the probe without the balloon based system has recently been developed for use with smaller lumens, most commonly the common bile duct and pancreatic duct with the hopes of better detection of pre-neoplastic and neoplastic lesions of the pancreaticobiliary system (30). VLE scans are reviewed manually based on an enhanced diagnostic algorithm and imaging characteristics. These include surface intensity greater than subsurface intensity, lack of layering, and presence of irregular and dilated glands/ducts (31-33).

Some of the proposed disadvantages to VLE include the training necessary to be proficient. Although studies are limited, early evidence suggests that the learning curve appears short (30). The learning curve after a brief training session for 31 novice clinicians showed that $71 \%$ were able to achieve competency during the 96-slide review, with half achieving competency at 65 slides $(16,34)$. Another drawback is high initial capital costs that generally limited its use to academic centers. Analysis of cost effectiveness is still ongoing given how new the technology is, but long term savings from faster imaging and more precise treatment leading to reduced subsequent procedures has been proposed (30). Safety is generally not a concern and it is typically well tolerated. Minor lacerations were reported in $2 \%$ of patients caused by a larger $25 \mathrm{~mm}$ balloon prior to its discontinuation $(16,35)$. One benefit is that it does not require a contrast infusion like CLE with the thought that it is at least safe, if not safer, compared to other modalities 


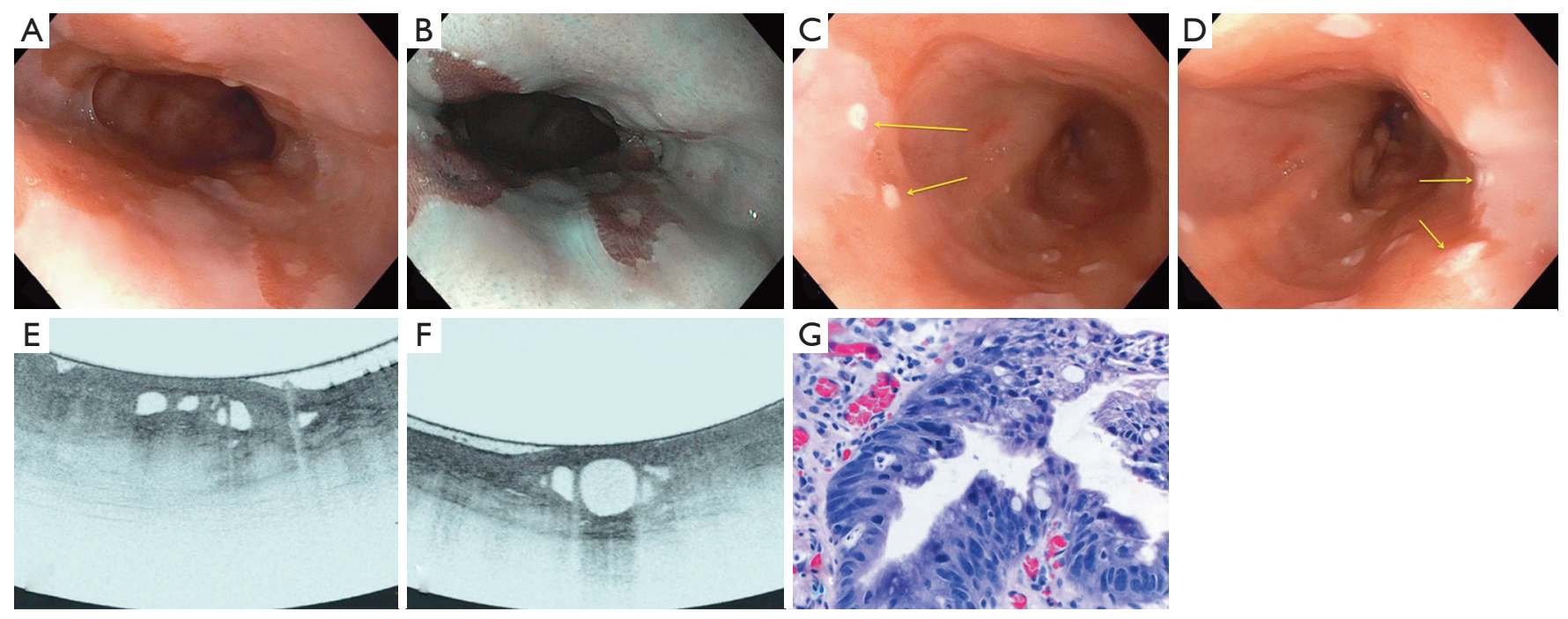

Figure 2 Endoscopic, VLE imaging and histologic results of evaluation for dysplasia in BE patient using 20-mm balloon. (A) Long-segment Barrett's esophagus without any focal abnormalities concerning for dysplasia; (B) the long-segment Barrett's esophagus examined on narrowband imaging without any focal abnormalities concerning for dysplasia; (C) laser marks (yellow arrows) placed over a concerning area within the Barrett's segment; (D) laser marks (yellow arrows) placed over a second area of concern within the Barrett's segment; (E) volumetric laser endomicroscopic image showing partial effacement and more than 5 atypical glands; (F) volumetric laser endomicroscopic image showing atypical glands and a dark surface intensity; $(\mathrm{G})$ histologic appearance of the first targeted area within the Barrett's segment showing highgrade dysplasia (H\&E, orig. mag. ×400). VLE, volumetric laser endomicroscopy; BE, Barrett's esophagus. (Figure used with permission) (39).

\section{What is the role of VLE in BE patients?}

A meta-analysis from Jain et al. suggested a high sensitivity (92.3\%) and negative predictive value $(83.3 \%)$ but a low specificity $(23.8 \%)$ for VLE in BE patients for dysplasia. The significance of these findings however is limited by a low cohort size (36). The 1,000 patients multicenter study found that as an adjunct modality, dysplasia diagnosis increased by $3 \%$ and the dysplasia diagnostic yield improved by $55 \%$ over standard practice (18). However, it is not uncommon to reveal Barrett's segments that lack layering or have atypical glands/ducts, but the biopsies do not show dysplasia (30). One study at Massachusetts General Hospital comparing VLE to pCLE showed the sensitivity and specificity of VLE for dysplasia detection at $86 \%$ and $88 \%$ respectively. A diagnostic accuracy of $87 \%$ was reported, significantly superior to that of pCLE (28). Another study found the sensitivity and specificity to be $90 \%$ and $93 \%$ respectively when using a computer related algorithm to automatically classify the images (37). Other studies with computer aided detection supported a high detection rate compared with manual reads (AUROC 0.95 vs. 0.81) (38). Future studies are needed to determine a negative predictive value which, if high, may support the use of VLE-target biopsies over the Seattle Protocol. An example of a VLE study is seen in Figure 2 (39).

\section{What is the role of VLE for BE endotherapy?}

In addition to surveillance, arguably the greatest clinical utility that VLE has is to guide treatment in BE with dysplasia. An accurate estimate of lesion size is important in determining resection approach. The rate of margin free resection in lesions of HGD dysplasia, IMC or early EAC has been estimated to be at about $50 \%$ under high definition WLE (40). VLE is able to identify mucosal lesions that are not seen under standard WLE. To increase the diagnostic precision, a laser guided device has been developed that marks the mucosal surface corresponding where both surface and subsurface abnormalities have been identified. One study compared incremental yield of dysplasia with VLE (24\%) compared to Seattle protocol biopsies (19.6\%) and random biopsies (5.7\%), where VLE with laser marked targets had a reported incremental yield of $33.7 \%$. The overall yield of dysplasia compared to the Seattle protocol was statistically higher $(\mathrm{OR}=2.1 ; \mathrm{P}=0.03)(32,39)$. The multi-center study found that endoscopists using VLE felt that it helped guide 
tissue acquisition in $70 \%$ of procedures (18).

\section{What is the role of VLE in BE for post treatment surveillance?}

Endoscopic therapy is generally highly effective, but recurrence rates can be as high as 20\% (41) and routine surveillance post therapy is recommended. VLE has been shown to be effective in 2 particular post treatment recurrences. One incidence being gastric cardia recurrence that is often not visible on endoscopy $(31,42)$. The other incidence is detecting sub-squamous intestinal metaplasia, or buried BE. VLE has been reported in detecting post ablation buried BE with a reported sensitivity of $80 \%$ and specificity of $100 \%$ (43). However, another small study of 17 post treatment patients found that VLE detected 13 with sub-squamous glandular structures (SGS) concerning for metaplasia, but only one was histologically found to be BE (44). This is likely due to lack of specific VLE features distinguishing buried BE and SGS.

\section{Conclusions}

VLE appears to be a useful tool to assist in screening, surveillance, and therapy when used as an adjunct to current standard practice modalities including endoscopy with WLE and Seattle protocol biopsies. VLE has specifically been shown to be effective at increasing the dysplasia diagnostic yield in detecting lesions missed by WLE and random biopsies. In addition, it has shown promise in better detection of recurrence in often missed areas post treatment. As this technology is still relatively new, further studies are needed to support routine use of VLE. Barriers include limited number of large sample studies, cost and training demands, and the lack of clear evidence supporting superiority over current less expensive and readily available methods.

\section{Acknowledgments}

None.

\section{Footnote}

Conflicts of Interest: The authors have no conflicts of interest to declare.

Ethical Statement: The authors are accountable for all aspects of the work in ensuring that questions related to the accuracy or integrity of any part of the work are appropriately investigated and resolved.

\section{References}

1. Hayeck TJ, Kong CY, Spechler SJ, et al. The prevalence of Barrett's esophagus in the US: estimates from a simulation model confirmed by SEER data. Dis Esophagus 2010;23:451-7.

2. Rex DK, Cummings OW, Shaw M, et al. Screening for Barrett's esophagus in colonoscopy patients with and without heartburn. Gastroenterology 2003;125:1670-7.

3. Van der Veen AH, Dees J, Blankensteijn JD, et al. Adenocarcinoma in Barrett's oesophagus: an overrated risk. Gut 1989;30:14-8.

4. Eckardt VF, Kanzler G, Bernhard G. Life expectancy and cancer risk in patients with Barrett's esophagus: a prospective controlled investigation. Am J Med 2001;111:33-7.

5. Wani S, Falk G, Hall M, et al. Patients with nondysplastic Barrett's esophagus have low risks for developing dysplasia or esophageal adenocarcinoma. Clin Gastroenterol Hepatol 2011;9:220-7.

6. American Gastroenterological Association, Spechler SJ, Sharma P, et al. American Gastroenterological Association medical position statement on the management of Barrett's esophagus. Gastroenterology 2011;140:1084-91.

7. Alvarez Herrero L, Pouw RE, van Vilsteren FG, et al. Safety and efficacy of multiband mucosectomy in 1060 resections in Barrett's esophagus. Endoscopy 2011;43:177-83.

8. Peters FP, Kara MA, Curvers WL, et al. Multiband mucosectomy for endoscopic resection of Barrett's esophagus: feasibility study with matched historical controls. Eur J Gastroenterol Hepatol 2007;19:311-5.

9. Pouw RE, van Vilsteren FG, Peters FP, et al. Randomized trial on endoscopic resection-cap versus multiband mucosectomy for piecemeal endoscopic resection of early Barrett's neoplasia. Gastrointest Endosc 2011;74:35-43.

10. Chevaux JB, Piessevaux H, Jouret-Mourin A, et al. Clinical outcome in patients treated with endoscopic submucosal dissection for superficial Barrett's neoplasia. Endoscopy 2015;47:103-12.

11. Hirasawa K, Kokawa A, Oka H, et al. Superficial adenocarcinoma of the esophagogastric junction: longterm results of endoscopic submucosal dissection. Gastrointest Endosc 2010;72:960-6.

12. Terheggen G, Horn EM, Vieth M, et al. A randomised 
trial of endoscopic submucosal dissection versus endoscopic mucosal resection for early Barrett's neoplasia. Gut 2017;66:783-93.

13. Manner H, May A, Kouti I, et al. Efficacy and safety of Hybrid-APC for the ablation of Barrett's esophagus. Surg Endosc 2016;30:1364-70.

14. van Lanschot JJ, Hulscher JB, Buskens CJ, et al. Hospital volume and hospital mortality for esophagectomy. Cancer 2001;91:1574-8.

15. Gross SA, Kingsbery J, Jang J, et al. Evaluation of Dysplasia in Barrett's Esophagus. Gastroenterol Hepatol (N Y) 2018;14:233-9.

16. Sharma P, Dent J, Armstrong D, et al. The development and validation of an endoscopic grading system for Barrett's esophagus: the Prague C\&M criteria. Gastroenterology 2006;131:1392-9.

17. Reid BJ, Blount PL, Feng Z, et al. Optimizing endoscopic biopsy detection of early cancers in Barrett's high-grade dysplasia. Am J Gastroenterol 2000;95:3089-96.

18. Smith MS, Cash B, Konda B, et al. Volumetric Laser Endomicroscopy and its application to Barretts esophagus: results from a 1000 patient registry. Diseases of the Esophagus 2019. doi: 10.1093/dote/doz029.

19. Shaheen NJ, Falk GW, Iyer PG, American College of Gastroenterology, et al. ACG Clinical Guideline: diagnosis and management of Barrett's esophagus. Am J Gastroenterol 2016;111:30-50.

20. Sami SS, Subramanian V, Butt WM, et al. High definition versus standard definition white light endoscopy for detecting dysplasia in patients with Barrett's esophagus. Dis Esophagus 2015;28:742-9.

21. Sharma P, Hawes RH, Bansal A, et al. Standard endoscopy with random biopsies versus narrow band imaging targeted biopsies in Barrett's oesophagus: a prospective, international, randomised controlled trial. Gut 2013;62:15-21.

22. Gupta N, Gaddam S, Wani SB, et al. Longer inspection time is associated with increased detection of high-grade dysplasia and esophageal adenocarcinoma in Barrett's esophagus. Gastrointest Endosc 2012;76:531-8.

23. Abrams JA, Kapel RC, Lindberg GM, et al. Adherence to biopsy guidelines for Barrett's esophagus surveillance in the community setting in the United States. Clin Gastroenterol Hepatol 2009;7:736-42.

24. Vennalaganti PR, Naag Kanakadandi V, Gross SA, et al. Inter-observer agreement among pathologists using wide-area transepithelial sampling with computerassisted analysis in patients with Barrett's esophagus. Am J Gastroenterol 2015;110:1257-60.
25. ASGE Technology Committee, Thosani N, Abu Dayyeh BK, et al. ASGE Technology Committee systematic review and meta-analysis assessing the ASGE Preservation and Incorporation of Valuable Endoscopic Innovations thresholds for adopting real-time imagingassisted endoscopic targeted biopsy during endoscopic surveillance of Barrett's esophagus. Gastrointest Endosc 2016;83:684-98.e7.

26. Longcroft-Wheaton G, Duku M, Mead R, et al. Acetic acid spray is an effective tool for the endoscopic detection of neoplasia in patients with Barrett's esophagus. Clin Gastroenterol Hepatol 2010;8:843-7.

27. Sharma P, Bergman JJ, Goda K, et al. Development and validation of a classification system to identify high-grade dysplasia and esophageal adenocarcinoma in Barrett's esophagus using narrow band imaging. Gastroenterology 2016;150:591-8.

28. Vakoc BJ, Shishko M, Yun SH, et al. Comprehensive esophageal microscopy by using optical frequency-domain imaging (with video). Gastrointest Endosc 2007;65:898-905.

29. Suter MJ, Gora MJ, Lauwers GY, et al. Esophageal-guided biopsy with volumetric laser endomicroscopy and laser cautery marking: a pilot clinical study. Gastrointest Endosc 2014;79:886-96.

30. Wolfsen HC. Volumetric Laser Endomicroscopy in Patients With Barrett Esophagus. Gastroenterol Hepatol (N Y) 2016;12:719-22.

31. Trindade AJ, Leggett CL, Chang KJ. Volumetric Laser endomicroscopy in the management of Barrett's esophagus. Curr Opin Gastroenterol 2017;33:254-60.

32. Evans JA, Poneros JM, Bouma BE, et al. Optical coherence tomography to identify intramucosal carcinoma and highgrade dysplasia in Barrett's esophagus. Clin Gastroenterol Hepatol 2006;4:38-43.

33. Leggett CL, Gorospe EC, Chan DK, et al. Comparative diagnostic performance of volumetric laser endomicroscopy and confocal laser endomicroscopy in the detection of dysplasia associated with Barrett's esophagus. Gastrointest Endosc 2016;83:880-8.e2.

34. Trindade AJ, Inamdar S, Smith MS, et al. Learning curve and competence for volumetric laser endomicroscopy in Barrett's esophagus using cumulative sum analysis. Endoscopy 2018;50:471-8.

35. Wolfsen HC, Sharma P, Wallace MB, et al. Safety and feasibility of volumetric laser endomicroscopy in patients with Barrett's esophagus (with videos). Gastrointest Endosc 2015;82:631-40.

36. Jain D, Fatama S, Jain S, et al. Volumetric Laser 
Endomicroscopy for Barrett's Esophagus- Looking at the Fine Print. J Gastrointestin Liver Dis 2017;26:291-97.

37. Swager AF, van der Sommen F, Klomp SR, et al. Computer-aided detection of early Barrett's neoplasia using volumetric laser endomicroscopy. Gastrointest Endosc 2017;86:839-46.

38. Sanghi V, Thota PN. Barrett's esophagus: novel Strategies for screening and surveillance. Ther Adv Chronic Dis 2019;10:2040622319837851.

39. Alshelleh M, Inamdar S, McKinley M, et al. Incremental yield of dysplasia detection in Barrett's esophagus using volumetric laser endomicroscopy with and without laser marking compared with a standardized random biopsy protocol. Gastrointest Endosc 2018;88:35-42.

40. Kamboj AK, Kahn A, Sawas T, et al. Outcome of endoscopic mucosal resection in Barretts esophagus determined by systematic quantification of epithelial glands using volumetric laser endomicroscopy. Gastrointest Endosc 2019;89:701-8.e1.

doi: $10.21037 / \operatorname{tgh} .2019 .11 .16$

Cite this article as: Houston T, Sharma P. Volumetric laser endomicroscopy in Barrett's esophagus: ready for primetime. Transl Gastroenterol Hepatol 2020;5:27.
41. Orman ES, Li N and Shaheen NJ. Systematic reviews and meta-analyses efficacy and durability of radiofrequency ablation for Barrett's esophagus: systematic review and meta-analysis. Clin Gastroenterol Hepatol 2013;11:1245-55.

42. Guthikonda A, Cotton CC, Madanick RD, et al. Clinical outcomes following recurrence of intestinal metaplasia after successful treatment of Barrett's esophagus with radiofrequency ablation. Am J Gastroenterol 2017;112:87-94.

43. Hatta W, Uno K, Koike T, et al. Feasibility of optical coherence tomography for the evaluation of Barrett's mucosa buried underneath esophageal squamous epithelium. Dig Endosc 2016;28:427-33.

44. Swager AF, Boerwinkel DF, de Bruin DM, et al. Detection of buried Barrett's glands after radiofrequency ablation with volumetric laser endomicroscopy. Gastrointest Endosc 2016;83:80-8. 